\title{
A structural comparison of lipopolysaccharide biosynthesis loci of Legionella pneumophila serogroup 1 strains
}

\author{
Markus Petzold ${ }^{1,2}$, Alexander Thürmer ${ }^{1}$, Susan Menzel ${ }^{1}$, Johan W Mouton ${ }^{2}$, Klaus Heuner ${ }^{3}$ and Christian Lück ${ }^{1 *}$
}

\begin{abstract}
Background: The lipopolysaccharide (LPS) is the major immuno-dominant antigen of all Legionella species including L. pneumophila. Its diversity is the basis for the classification of L. pneumophila into serogroups and monoclonal subgroups and is thought to be involved in strain specific virulence. The understanding of the genetic basis of the LPS-antigen is incomplete. Thus, we analyzed the genetic locus involved in LPS-biosynthesis of L. pneumophila serogroup 1 (Sg1) strains with the focus on strain specific gene composition.

Results: The LPS-biosynthesis loci of 14 L. pneumophila Sg1 strains comprise two distinct regions: A 15 kb region containing LPS-biosynthesis genes that can be found in all L. pneumophila strains and a Sg1-specific $18 \mathrm{~kb}$ region. The $15 \mathrm{~kb}$ region is highly conserved among $\mathrm{Sg} 1$ strains as reflected by high homologies of single ORFs and by a consistent ORF arrangement. In contrast, the $\mathrm{Sg} 1$ specific $18 \mathrm{~kb}$ region is variable and partially disrupted by phage related genes. We propose that the region spanning from ORF 6 to ORF 11 of the Sg1-specific region is likely involved in late LPS-modification. Due to the high variability of this small region and various combinations of single ORFs within this region a strain specific LPS-structure could be synthesized including modifications of legionaminic acid derivates.
\end{abstract}

Conclusions: Our data clearly demonstrate that the gene structure of the LPS-biosynthesis locus of L. pneumophila Sg1 strains show significant interstrain variability. These data can be used for further functional analysis of the LPS synthesis to understand pathogenesis and reactivity with monoclonal antibodies. Moreover, variable but strain specific regions can serve as basis for the development of novel genotyping assays.

Keywords: Legionella pneumophila, Lipopolysaccharide, Locus organization, Monoclonal antibody typing

\section{Background}

Legionella pneumophila is one of 56 described species belonging to the genus Legionella of the family Legionellaceae [1]. These Gram-negative bacteria are ubiquitous inhabitants of natural and manmade aquatic environments where they survive parasitically in protozoa like amoeba $[2,3]$ and in community structures such as biofilms [4,5]. Additionally, Legionella can infiltrate the human lung via inhaled aerosols $[3,6]$ and subsequently infect alveolar macrophages [7] which frequently cause a potential fatal pneumonia termed Legionnaires' disease (LD) [8]. L. pneumophila

\footnotetext{
* Correspondence: christian.lueck@tu-dresden.de

'Institute of Medical Microbiology and Hygiene, Faculty of Medicine 'Carl Gustav Carus', University of Technology Dresden, Fetscherstraße 74, Dresden D-01307, Germany

Full list of author information is available at the end of the article
}

strains belonging to the serogroup 1 (Sg1) were predominantly reported in LD cases, especially in community acquired and travel-associated cases $[9,10]$.

Lipopolysaccharide (LPS) is the major immunodominant antigen of all Legionella species including L. pneumophila [11]. It is the main component recognized by patient's sera and by diagnostic assays in urinary antigen detection [12]. The LPS molecule possesses a high degree of diversity and thereby provides the basis for the classification of $L$. pneumophila into serogroups and subgroups by monoclonal antibodies (mAb) [13-15]. Sg1 strains are subdivided into nine $\mathrm{mAb}$-subgroups using the Dresden monoclonal antibody panel (Table 1) [16].

The structural characterization of LPS of L. pneumophila identified several specific chemical attributes which differs it from the LPS molecules of other Gram-negative 


\begin{tabular}{|c|c|c|c|c|c|c|c|c|}
\hline $\begin{array}{l}\text { Monoclonal } \\
\text { antibody subgroup }\end{array}$ & $8 / 5$ & $3 / 1$ & 3 & $8 / 4$ & $10 / 6$ & $20 / 1$ & $26 / 1$ & $30 / 4$ \\
\hline Philadelphia & + & + & - & + & - & - & - & - \\
\hline Allentown/France & + & + & - & - & - & - & - & $+/-^{a}$ \\
\hline Benidorm & + & + & - & - & - & + & - & - \\
\hline Knoxville & + & + & + & - & - & - & - & - \\
\hline OLDA & + & - & - & + & - & $+/-^{a}$ & + & $+/-^{a}$ \\
\hline Oxford & + & - & - & + & - & - & - & $+/-^{a}$ \\
\hline Heysham & + & - & + & - & - & - & - & - \\
\hline Camperdown & + & - & - & - & - & - & + & + \\
\hline Bellingham & + & - & - & - & + & + & + & + \\
\hline
\end{tabular}

${ }^{a}$ Variable results with different strains.

bacteriareviewd in [17]. Particularly the $O$-antigen homopolymer structure consists of an unusual residue, 5acetamidino-7-acetamido-8-O-acetyl-3, 5, 7, 9-tetradesoxy - $D$-glycero- $D$-galacto-nonulosonic acid (legionaminic acid) and its derivates [18-20].

A central step in understanding the correlation of the LPS structure and pathogenesis of L. pneumophila was the description of the genetic background of LPS molecules by Lüneberg and colleagues [21]. More precisely, a genetic locus composed of at least 28 open reading frames (ORF) is essential in LPS core oligosaccharide biosynthesis and LPS $O$-chain biosynthesis. The genes of this 31-36 kb cluster have characteristic functions required for the synthesis, transport, translocation and modification of LPS components. The lag-1 gene of this biosynthesis locus encodes for an $O$-acetyltransferase which is responsible for the 8-O-acetylation of legionaminic acid [22]. Strains carrying a functional lag-1 synthesize an LPS epitope that reacts with the mAb 3/1 (initially named mAb 2 [23]) of the Dresden monoclonal antibody panel. This epitope is assumed to contribute to an increased virulence $[22,24]$ since $\mathrm{mAb} 3 / 1^{+}$strains represent the most prominent subgroup of clinical $L e$ gionella isolates. In contrast, strains lacking lag-1 carry mainly deacetylated LPS molecules. These mAb 3/1 strains comprise only a small number of clinically identified L. pneumophila strains in immunocompetent patients $[9,10]$.

Besides the mAb 3/1 specific $O$-acetylation of the legionaminic acid epitope, to date it remains elusive how strain specific mAb-reactivities can be explained. Increased understanding of the genetic background and structural LPS properties of the different Sg1 strains could help to comprehend subgroup distributions among clinical and environmental isolates [9,16,25-27] and would deliver more insight in the role of LPS in the L. pneumophila life cycle.
To achieve this goal, we analyzed the LPS-biosynthesis loci of at least one member of each mAb-subgroup (excluding mAb-subgroup Oxford) of the L. pneumophila Sg1. In this study we focused on the genetically composition of the loci and putative genotype-phenotype correlations according to the Dresden panel of mAbs.

\section{Results and discussion}

Two regions within the LPS-biosynthesis locus

To gain insight into the genetic composition and arrangement of the LPS biosynthesis locus we analyzed the loci of $14 \mathrm{~L}$. pneumophila Sg1 strains. The strains represent members of all $\mathrm{mAb}$-subgroups that can be distinguished by the Dresden monoclonal antibody panel (Table 1) besides the extremely rare mAb-subgroup Oxford. The LPS biosynthesis loci of five strains were newly sequenced for this study. These were: Camperdown 1 and Heysham 1 of the rarely found subgroups of the same name $[9,25]$ and the strains Uppsala 3, Görlitz 6543 and L10/23. Eight LPS biosynthesis loci were obtained from complete genomes that have been published previously. Furthermore, for strain RC1 (mAb subgroup OLDA) the biosynthesis locus was available as well (Table 2).

The LPS-biosynthesis locus of each of the analyzed L. pneumophila Sg1 strains contained at least 28 ORFs and ranged in size from 30,644 bp (strain Lorraine) to 35,888 bp (strain 130b) with an average locus size of 33,398 bp respectively. The average ORF size within the locus was approximately $1 \mathrm{~kb}$. The complete LPSbiosynthesis locus had a slightly lower \% GC content ( $35 \%)$ than the adjacent regions $(\sim 38 \%)$ and the total genome $(\sim 38.5 \%)$, respectively.

Structural and comparative analysis of the loci confirmed a highly conserved $15 \mathrm{~kb}$ region from wecA (ORF 14) to $\operatorname{lpg} 0748$ (ORF 28) according to the Philadelphia genome as shown previously [34]. Additionally, all ORFs within this region were consistently orientated into the same direction (Figure 1A and B).

A second region within the locus of $18 \mathrm{~kb}$ in size is spanning from lpg0779 (ORF 1) to lpg0764 (ORF 13). Here, the structural composition and the orientation of ORFs as well as the total amount of putative ORFs was less conserved. Interestingly, many transposases and phage related genes were present in 8 strains (Figure 1A).

The heterogeneous nature of the $18 \mathrm{~kb}$ region and the extremely high conserved $15 \mathrm{~kb}$ region found in our study are largely in agreement with earlier results. These proposed to separate the locus into a Sg1 specific and a L. pneumophila specific region [34,35]. Microarray analysis of Sg1 and non-Sg1 strains have identified a $13 \mathrm{~kb}$ region (ORF 16-28) which is present in all L. pneumophila strains and a $20 \mathrm{~kb}$ region (ORF 1-15) generally found in all Sg1 strains [34]. The two regions 
Table 2 LPS biosynthesis loci obtained from sequenced genomes of L. pneumophila Sg1 strains

\begin{tabular}{|c|c|c|c|}
\hline Strain & mAb subgroup & Accession no. & Reference \\
\hline Alcoy 2300/99 & Knoxville & GenBank: NC_014125.1 & {$[28]$} \\
\hline Corby & Knoxville & GenBank: NC_009494.2 & [29] \\
\hline L10/23 (Ulm) & Knoxville & EMBL: HF545881 & this study \\
\hline Uppsala $3^{*}$ & Knoxville & EMBL: HE980445 & this study \\
\hline Paris & Philadelphia & GenBank: NC_006368.1 & [30] \\
\hline Philadelphia 1 & Philadelphia & GenBank: NC_002942.5 & [31] \\
\hline HL 06041035 & Bellingham & EMBL: FQ958211 & [32] \\
\hline Görlitz 6543* & Bellingham & EMBL: HF678227 & this study \\
\hline Camperdown $1^{*}$ & Camperdown & EMBL: HE980447 & this study \\
\hline Heysham $1^{*}$ & Heysham & EMBL: HE980446 & this study \\
\hline 130b (Wadsworth) & Benidorm & EMBL: FR687201 & [33] \\
\hline Lens & Benidorm & GenBank: NC_006369.1 & {$[30]$} \\
\hline Lorraine & Allentown & EMBL: FQ958210 & {$[32]$} \\
\hline $\mathrm{RC}^{*}$ & OLDA & EMBL: AJ277755 & [21] \\
\hline
\end{tabular}

* only LPS biosynthesis locus sequenced.

were defined based on the LPS-biosynthesis loci of the Sg1 strain Paris [30].

To determine the putative breakpoint between both regions is difficult. However, based on our analysis of the structural composition we would rather separate the LPS biosynthesis locus between lpg0763 (ORF 13) and wecA (ORF 14). This is in agreement with recent data, since the genes wecA (ORF 14) and galE (ORF 15) were demonstrated to be present in non-Sg1 strains with lower amino acid similarities when compared to Sg1 strains $(55-61 \%)$ [35].

The initially mentioned ORF 13 is located next to the breakpoint region. In total, four different types of ORFs were found in the analyzed region of $\mathrm{Sg} 1$ strains here named ORF 13-a, -b, -c and -A. In each of the strains Lens, 130b, HL 06041035 and Görlitz 6543 two ORFs were found. These strains carried a putative conserved protein of unknown function (here referred to as ORF 13-A). A transposase-disrupted ORF 13-A was present in strain 130b (Figure 1A). Additionally, the strains carried an ORF which shared features of the radical S-adenosylmethionine (SAM) superfamily (CDD: cd01335) named ORF 13-c (Additional file 1: Table S2). Interestingly, all these strains lacked the ORF 12. However, even though the strain Lorraine lacked ORF 12 as well, it carried only a single ORF 13-A variant.

A distinct ORF of unknown function with amino acid similarity to ORF 13 -A of only $38 \%$, here named ORF 13 -a, was present in the remaining strains with the exceptions of a truncated form in strains RC1, Philadelphia 1 and Paris. Philadelphia 1 and Paris shared high similarities with ORF 13-a but a deletion led to a frame shift resulting into three smaller fragments (pooled as ORF 13-b) (Table 3).

\section{A conserved region found in all serogroup 1 strains}

Within the conserved region several genes were found which are proposed to be involved in the biosynthesis of the highly acetylated core region which is composed of mannose, N-acetyl-glucosamine (GlcNAc), N-acetylquinovosamine (QuiNAc) and rhamnose residues [19]. A vast number of ORFs, more specifically ORF 21 through 25 and 28, were recently reported to facilitate the biosynthesis of the repetitive legionaminic acid residues of the $O$-antigen [18,36]. The pyrodoxal-phosphate dependent aminotransferase (ORF 21), the acetyltransferase neuD (ORF 22) and a dehydratase (lpg0966) located outside of the locus are likely to synthesize the precursor molecule of legionaminic acid, UDP- $N, N^{\prime}$-diacetylbacillosamine (UDPBac2Ac4Ac) [37]. Contradictory to our findings, functions of the neuD products are described highlighting that the acetyltransferase is involved in Lag-1-independent $O$ acetylation of few legionaminic acid residues close to the LPS-core of L. pneumophila $[21,38,39]$. Furthermore, the adjacent genes encoding for NeuC (ORF 23), NeuB (ORF 24) and NeuA (ORF 25) were recently identified to be involved in the subsequent biosynthetic processes converting UDP-Bac2Ac4Ac into CMP-5,7-diacetamido-3,5,7, 9-tetradeoxy-D-glycero-D-talo-nonulosonic acid (CMPLeg5Ac7Ac) [36]. Moreover, the ORF 28 is homologous to the ptmG gene of Campylobacter jejuni (Cj1324) which converts the CMP-Leg5Ac7Ac residue to CMP-5acetamidino-7-acetamido-3,5,7,9-tetradeoxy-D-glycero-D- $t$ alo-nonulosonic acid (CMP-Leg5Am7Ac) [40], the dominant residue of the $\mathrm{O}$-antigen of non-Sg1 strains of L. pneumophila [41]. A functional correlation of the ORFs of this region is supported by recent transcriptomic data of strain Paris in which the ORFs 21-17 and 28-22 were transcribed as operons [42]. Since all analyzed Sg1 strains 


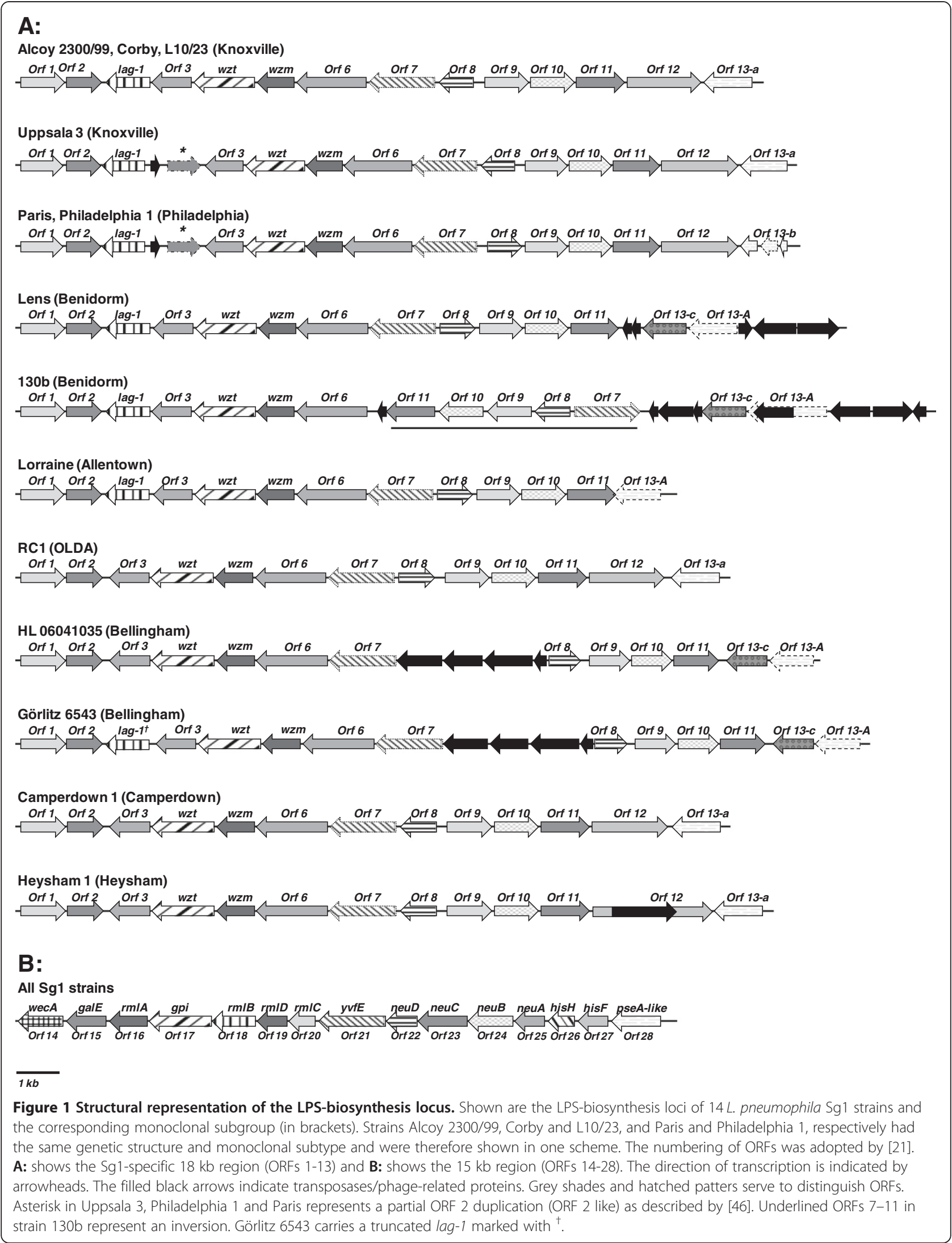


Table 3 Amino acid similarity of the L. pneumophila Sg1 specific LPS-biosynthesis region from Ipg0769-Ipg0761 (ORF 1 - ORF 15) of strain Philadelphia 1 to other Sg1 strains

\begin{tabular}{|c|c|c|c|c|c|c|c|c|c|c|c|c|c|c|}
\hline \multicolumn{15}{|c|}{ Amino acid similarity [\%] ${ }^{*}$} \\
\hline \multicolumn{2}{|c|}{ Gene name of $L$. pneumophila } & \multirow{2}{*}{$\begin{array}{c}\text { Philadelphia\# } \\
\text { Paris }\end{array}$} & \multicolumn{4}{|c|}{ Knoxville\# } & \multicolumn{2}{|c|}{ Benidorm\# } & \multicolumn{2}{|c|}{ Bellingham\# } & \multirow{2}{*}{$\begin{array}{c}\text { Allentown\# } \\
\text { Lorraine }\end{array}$} & \multirow{2}{*}{$\begin{array}{c}\text { OLDA\# } \\
\text { RC1 }\end{array}$} & \multirow{2}{*}{$\begin{array}{l}\text { Camperdown\# } \\
\text { Camperdown } 1\end{array}$} & \multirow{2}{*}{$\begin{array}{l}\text { Heysham\# } \\
\text { Heysham1 }\end{array}$} \\
\hline \multicolumn{2}{|c|}{ Sg1 strain Philadelphia 1} & & 2300/99 Alcoy & Corby & Uppsala 3 & Ulm & $130 \mathrm{~b}$ & Lens & HL 06041035 & Görlitz 6543 & & & & \\
\hline Ipg0761 (galE) & ORF 15 & 100 & 100 & 100 & 100 & 100 & 97.1 & 96.0 & 99.8 & 99.8 & 99.8 & 100 & 100 & 100 \\
\hline $\operatorname{lpg} 0762$ (wecA) & ORF 14 & 100 & 99.5 & 99.5 & 99.5 & 99.5 & 93.4 & 93.1 & 93.7 & 93.7 & 93.4 & 98.8 & 99.5 & 99.5 \\
\hline Ip/0803 ${ }^{\mathrm{A}}$ & ORF 13 & - & 40.3 & 40.3 & 40.3 & 40.3 & trans. $^{c}$ & 100 & 98.2 & 98.2 & 96.6 & 41.8 & 40.3 & 40.3 \\
\hline $\operatorname{lpg} 0765$ & ORF 12 & 100 & 98.6 & 98.7 & 98.6 & 98.6 & - & - & - & - & - & 98.7 & 98.6 & trans. $^{c}$ \\
\hline Ipg0766 & ORF 11 & 100 & 96.6 & 96.6 & 96.6 & 96.6 & 93.2 & 93.2 & 93.7 & 93.7 & 93.1 & 96.6 & 96.6 & 96.6 \\
\hline Ipg0767 & ORF 10 & 100 & 96.2 & 96.2 & 96.2 & 96.2 & 96.6 & 97.1 & 98.9 & 98.9 & 97 & 95.6 & 96.2 & 96.2 \\
\hline Ipg0768 & ORF 9 & 100 & 30.6 & 30.6 & 30.6 & 30.6 & 98.4 & 99 & 99 & 99 & 98.9 & 99.4 & 30.6 & 30.6 \\
\hline Ipg0769 & ORF 8 & 100 & 31 & 31 & 31 & 31 & 97.9 & 97.4 & 98.4 & 98.4 & 97.4 & 100 & 31 & 31 \\
\hline Ipg0770 & ORF 7 & 100 & 90.6 & 90.6 & 90.6 & 90.6 & 32 & 31.9 & 31.9 & 31.9 & 99.8 & 99.9 & 90.6 & 90.6 \\
\hline Ipg0771 & ORF 6 & 100 & 38.8 & 38.7 & 38.7 & 38.7 & 38.8 & 99.1 & 100 & 100 & 38.8 & 38.6 & 99.1 & 38.7 \\
\hline Ipg0772 (wzm) & ORF 5 & 100 & 100 & 100 & 100 & 100 & 100 & 100 & 100 & 100 & 100 & 100 & 100 & 100 \\
\hline Ipg0773 (wzt) & ORF 4 & 100 & 99 & 99.6 & 100 & 100 & 100 & 99.6 & 100 & 99.5 & 99 & 99.8 & 100 & 100 \\
\hline Ipg0774 & ORF 3 & 100 & 91.6 & 86.4 & 98.7 & 92.1 & 89 & 86.4 & 100 & 86.4 & 91.6 & 99.5 & 99.8 & 99.8 \\
\hline $\operatorname{lpg} 0775^{\mathrm{a}}$ & & 100 & & - & 100 & - & - & - & - & - & - & - & - & - \\
\hline $\operatorname{lpg} 0776^{\mathrm{b}}$ & & 100 & - & - & 100 & - & - & - & - & - & - & - & - & - \\
\hline Ipg0777 (lag-1) & & 100 & 96.8 & 94.9 & 100 & 96.8 & 94.9 & 94.9 & - & $94.7^{\dagger}$ & 96.8 & - & - & - \\
\hline Ipg0778 & ORF 2 & 100 & 97.9 & 97.4 & 100 & 97.7 & 97.4 & 97.4 & 99.6 & 96.5 & 97.9 & 98.9 & 98.7 & 98.7 \\
\hline Ipg0779 & ORF 1 & 100 & 99.8 & 99.1 & 99.8 & 99.8 & 98.9 & 98.9 & 100 & 98.9 & 99.8 & 99.4 & 99.8 & 99.8 \\
\hline
\end{tabular}

* Determined by UPGMA clustering method based on multiple sequence alignment

* Determined by UPGMA clustering method based on multiple sequence alignment.

a Partial duplication of ORF 2 (Ipg0778).

b, c Transposase; transposase disrupted.

${ }^{+}$Lag-1 of Görlitz 6543 has no functional start codon.
Latrans

Underlined numbers indicate different clusters of corresponding ORF (see also Figure 2).

The highly conserved $15 \mathrm{~kb}$ region (ORF14 - ORF 28) is not completely shown and only reflected by WecA and GalE. 
and a broad number of non-Sg1 strains carry ORF 28 $[35,43,44]$ it can be assumed that CMP-Leg5Am7Ac is a common residue of the L. pneumophila LPS molecule which might subsequently become modified in a mAbsubgroup or even strain specific manner.

\section{Three clusters of the 0 -acetyltransferase Lag- 1}

A well examined phenotype variation is linked to the presence and absence of the lag-1 gene. Lag-1 encodes for an $O$-acetyltransferase that conferred reactivity with $\mathrm{mAb} 3 / 1$ and is exclusively found in Sg1 strains. Our results revealed three clusters of the lag-1 genes, although without any detectable relation to the mAb-subgroup switch which supports recent findings [45] (Figure 2A). Lag-1 was previously reported to be involved in $\mathrm{mAb}$ subgroup switches of different strains. However, this was generally due to gene deletion or loss-of-function mutations of lag-1 [46-49]. Complete and functional lag-1 genes were present in all $\mathrm{mAb} 3 / 1^{+}$strains and were absent in all $\mathrm{mAb} 3 / 1^{-}$strains. Besides that, the Philadelphia subgroup strains (Philadelphia 1 and Paris) as well as the Knoxville-subgroup strain Uppsala 3 carried a transposase and a partial duplication of ORF 2 adjacent to lag-1. Bernander et al. reported the region from ORF 2 to ORF 3 as unstable [46]. Looping out of the intermediate located lag-1 gene is assumed to be a potential consequence. Under in vitro conditions the deletion of the lag-1 gene occurred at with frequency of $10^{-6}$ to $10^{-7}$ (C. Lück, unpublished results). Detailed analysis of the region from ORF 2 to ORF 3 including lag-1 of these strains revealed remarkably high similarities of Uppsala 3 to the Philadelphia-subgroup strains Philadelphia 1 and Paris (>98-100\%) whereas the remaining Knoxville-subgroup strains clustered in a different group (Table 3; Figure 2A). The high similarity of this $4 \mathrm{~kb}$ region between strain Uppsala 3 and the strains Paris and Philadelphia 1 may indicate horizontal gene transfer of this region. However, this had no impact on the specific mAb reactivity for all other analyzed Knoxville-subgroup strains. Horizontal gene transfer between strain Paris and Philadelphia 1 was recently reported for a large genome fragment which also harbored the LPS biosynthesis locus [32]. These observations are confirmed by our results since the LPS biosynthesis loci of both strains are almost identical in composition, arrangement and amino acid similarity (Additional file 1: Table S2). A truncated lag-1 gene was found in the strain Görlitz 6543 (mAb-subgroup Bellingham) as recently reported [49]. The whole gene is present but carries a mutated start codon. Since Görlitz 6543 showed no reactivity with mAb 3/1 it was assumed that the mutation significantly impairs the production of a functional $O$-acetyltransferase. Phylogenetic analysis showed $99.9 \%$ amino acid similarity of Görlitz 6543 to

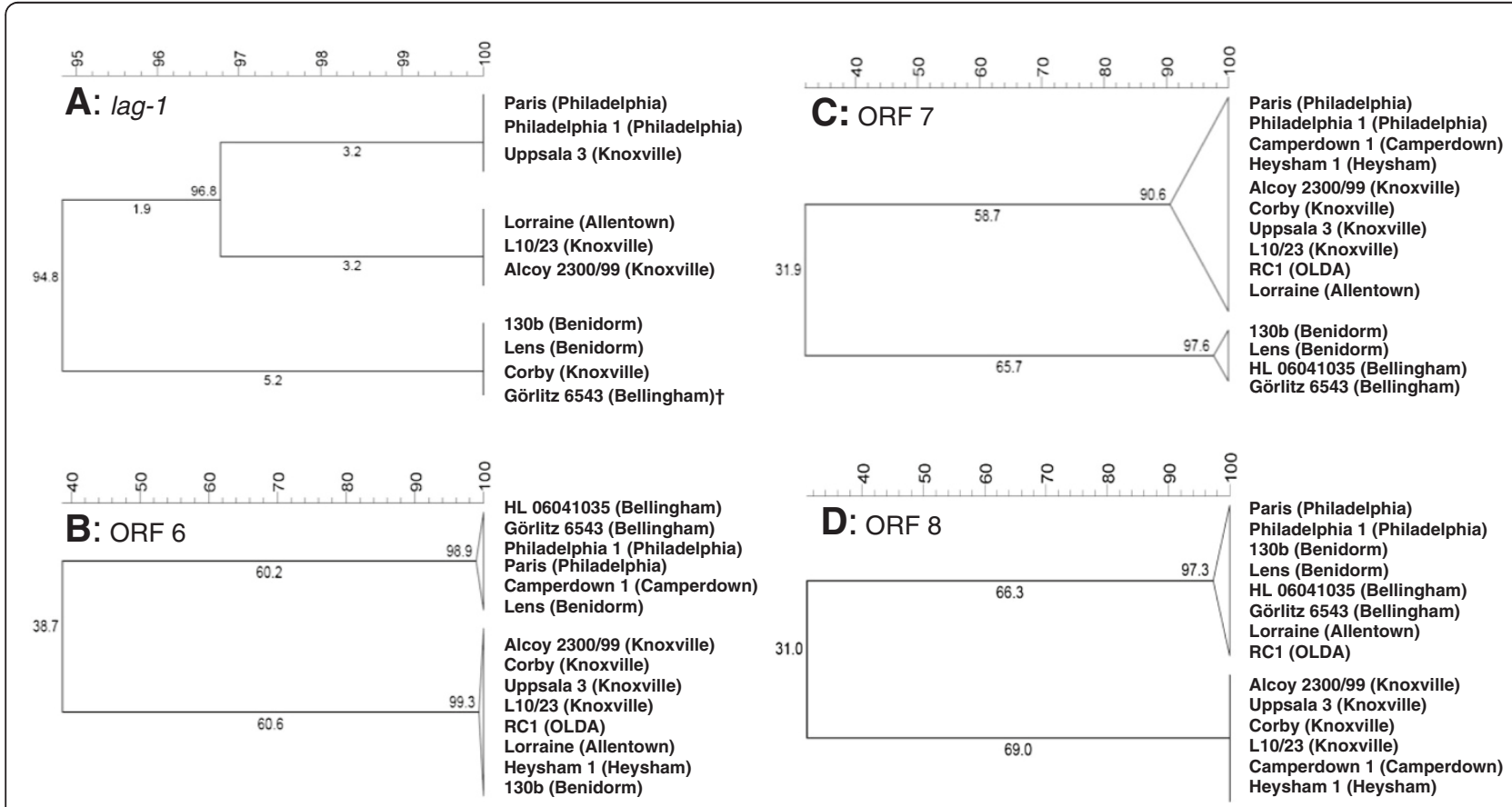

Figure 2 Dendrogram of variable ORFs. Multiple amino acid based cluster analysis using UPGMA (BioNumerics, Applied Maths NV, Belgium). The phylogenetic trees of gene lag-1 and of the ORFs 6,7 and 8 are shown. ORF 9 is identical to the phylogenetic tree of ORF 8 and is therefore not shown. Similarity values and branch distances were depicted in percentages [\%]. The strain-specific mAb-subgroup is indicated in brackets. The mutated start codon of lag-1 of Görlitz 6543 was neglected for similarity analysis and is indicated with ${ }^{\dagger}$. 
Corby (mAb-subgroup Knoxville), 130b and Lens (both mAb-subgroup Benidorm) (Figure 2A).

\section{ABC-transporter genes wzt and wzm as Sg1-specfic marker region}

Noticeable conserved genes within the heterogenic region were $w z t$ (ORF 4) and wzm (ORF 5) which are almost identical among all analyzed Sg1 strains (Figure 1A, Table 3). Wzm encodes for a protein containing a transmembrane domain while $w z t$ encodes for a nucleotide binding domain of an $\mathrm{ABC}$ transporter system which mediates the $\mathrm{O}$-antigen translocation across the inner membrane [50]. Recently, both genes were evaluated as marker genes for PCR based discrimination between L. pneumophila Sg1 and non-Sg1 strains [35]. The ABC transporter-dependent $O$-antigen pathway interacts with WecA (ORF 14), an UDP-GlcNAc-1transferase which initiates $O$-chain biosynthesis at the cytoplasmic site of the inner membrane [50]. The low amino acid similarity of WecA between Sg1 and nonSg1 that was described recently combined with the absence of $w z m$ and $w z t$ in non-Sg1 genomes [35] indicate a different $\mathrm{O}$-chain biosynthesis mechanism for non-Sg1 strains than found in Sg1 strains.

\section{ORF 6 through 11 involved in 0 -antigen modification}

The most variable region within the Sg1-specific region in terms of low similarities on the amino acid level and the diverse arrangement of single ORFs was found from ORF 6 to ORF 11. The strains of mAb-subgroup Benidorm 130b and Lens were almost identical regarding the amino acid similarities of the single ORFs within the Sg1-specific region. Interestingly, strain 130b carried a large inverted fragment containing ORF 7 to ORF 11 (Figure 1A). This region was surrounded by transposases suggesting their potential contribution to the inversion. Since the strain 130b showed no altered reactivity pattern using the Dresden panel compared to other Benidorm strains it could be stated that the inversion had no detectable effect on the LPS phenotype detected by monoclonal antibodies.

The adjacent ORF 6 showed a high degree of variability between L. pneumophila Sg1 strains represented by two clusters of low amino acid similarity $(<39 \%)$ (Figure 2B). Interestingly, the two analyzed strains of the mAb-subgroup Benidorm, 130b and Lens, cluster into two distinct groups. This either indicates that the product of ORF 6 has probably no effect on the LPS structure of strains of the same monoclonal subgroup or that it has the same function despite low similarity.

However, ORF 6 products might be involved in the establishment of a mAb-subgroup discriminating epitope. More precisely, only the mAb-subgroups Heysham and Knoxville react with $\mathrm{mAb} 3$. This indicates a similar epitope which in turn could possibly be traced back to specific ORFs within the Sg1-specific region. However, strains of both mAb-subgroups were highly homologous regarding the whole LPS-biosynthesis with the exception of lag-1 which is present in Knoxville strains. (Figure 2B, Table 3). In addition, the strain Camperdown 1, not reacting with $\mathrm{mAb} 3$, carried a very similar LPSbiosynthesis locus as Heysham 1 and the Knoxville strains. However, it is the single ORF 6 in which Camperdown 1 clusters differently to Heysham 1 . It can be assumed that the combination of ORF 6 to 9 which is exclusively found in Knoxville and Heysham strains leads to reactivity with mAb 3. Another ORF 6 as found in the genetically very similar strain Camperdown 1 could alter the LPS epitope and is thereby not recognized by mAb 3 . Furthermore, the mAb 3 epitope was not influenced by $O$-acetylation of the legionaminic acid residue since the Knoxville strains were $\mathrm{mAb} \mathrm{3/1}{ }^{+}$and carried the lag-1 gene whereas the strain Heysham 1 is negative for both markers.

\section{Modification of legionaminic acid in transposon mutants} Two additional ORFs, ORF 8 and ORF 9, within in the highly variable region from ORF 6 to ORF 11 are most likely involved in $\mathrm{O}$-antigen modification. The genetic nature of the ORF 8 products displayed two different clusters which was comparable to the clustering of ORF 9. Both clusters share poor amino acid similarities of 31\% (ORF 8) and 30.7\% (ORF 9) (Table 3, Figure 2D). These differences in amino acid similarity were also reflected by the ORF orientation. Both ORFs were orientated into opposite directions in strains of the mAbsubgroups Knoxville, Camperdown and Heysham which form a separate cluster in both ORFs (Figure 1A). For the remaining mAb-subgroups (Philadelphia, Allentown, Benidorm, Bellingham and OLDA) the ORFs are oriented into identical directions. In silico analysis of these loci predicted a five-gene operon from ORF 8 to ORF 12 suggesting a coupled functional entity [51]. These strains were also grouped into a single cluster. However, recent transcriptomic data obtained from strain Paris revealed a four-gene operon which lacks ORF 8 [42]. For all strains regardless of the distance in the phylogenetic tree BLASTP predicted a methyltransferase function for ORF 8 [48,52] and a siliac acid synthetase function (neuB family) for ORF 9 [21].

It is reported that the putative methyltransferase encoded by ORF 8 is responsible for $N$-methylation of the 5-acetimydyol amino group of the legionaminic acid $[48,52]$. This is supported by studies on the legionaminic acid pathway of Campylobacter. The ptmH gene (Cj1325) of C. jejuni is a homologue of ORF 8 of the Knoxville, Camperdown and Heysham subgroup cluster (Figure 2D) [40]. The ptmH product catalyzes the 
modification of CMP-Leg5Am7Ac to the $N$-methylated residue CMP-5-acetimidoyl ( $N$-methyl) amino-7-acetami do-3,5,7,9-tetradeoxynon-2-ulosonic acid (CMP-Leg5Am $\mathrm{NMe} 7 \mathrm{Ac}$ ), the main residue of the $\mathrm{Sg} 1 \mathrm{O}$-antigen. Disruption of ORF 8 in the Bellingham-subgroup strain Görlitz 6543 led to loss-of-reactivity with the Bellingham-subgroup specific mAb 10/6 and mAb 20/1 and resulted in a mAb-subgroup switch from subgroup Bellingham to Camperdown. In similar mutants of the $\mathrm{mAb} 3 / 1^{+}$strain $130 \mathrm{~b}$ the reactivity with mAb $20 / 1$ was also lost when ORF 8 or ORF 11 was disrupted leading to a switch from mAb-subgroup Benidorm to Allentown. The wild type strains $130 \mathrm{~b}$ and these mutants did not react with mAb10/6. This supported the assumption that the mAb 3/1-specific epitope generated by the $O$-acetyltransferase Lag- 1 masks the $N$-methyl group and hinders binding of mAb 10/6 [48]. This is in agreement with earlier observations which reported a correlation between ORF 8 and $N$-methylated legiona minic acid residues for the mAb $3 / 1^{-}$strain $\mathrm{RC} 1$ [52]. However, the fact that mutants of both strains, $130 \mathrm{~b}$ and Görlitz 6543, lost the reactivity with mAb 20/1, indicated that ORF 8 and/or ORF 11 are also involved in the generation or modification of another epitope which is not blocked by the $O$-acetyl group.

To find putative ORF candidates, next to ORF 8, that are responsible for synthesis or modification of the common epitope bound by mAb 20/1, we looked for similar but unique ORFs within the Sg1-specific region of Bellingham- and Benidorm-subgroup strains. Phylogenetic analyses identified ORF 7 as a putative subgroup discriminating gene since the mAb-subgroups Benidorm and Bellingham clustered in specific separate group when compared to the other mAb-subgroups (Figure $2 \mathrm{C}$ ). The presence of two different ORF 7 variants is in agreement with recent results obtained by subgroup specific PCR amplification [49].

\section{Conclusions}

Characterization of the LPS-biosynthesis loci of L. pneumophila Sg1 strains revealed two mayor regions: A Sg1-specific region of $18 \mathrm{~kb}$ and a conserved $15 \mathrm{~kb}$ region containing genes found in Sg1 and non-Sg1 strains. The conserved region carries genes involved in outer core and $O$-chain biosynthesis of LPS molecules.

The variable and heterogeneous Sg1-specific region raised questions concerning the genetic basis for subgroup specific mAb-reactivity. Switches from one monoclonal subtype to another in transposon induced mutants gave a first indication for the function of different gene products. The most variable region from ORF 6 to ORF 11 is likely involved in $O$-antigen modifications and could deliver more insight in the strain specific LPS structures and more important, in strain specific virulence. The ORFs within this region could act in a pathway-like manner explaining the broad variability of the LPS molecule among the Sg1 strains. Furthermore, it is also not excluded that each ORF of this region has an own function in the late modification of legionaminic acid derivates which could be regulated in a life cycle or growth phase-depended way. Further studies using specific mutation in these ORFs, mRNA assays and chemical analysis are required in order to elucidate the role of different genes in the synthesis of the subgroup specific structures in different strains.

\section{Methods \\ Phenotypic and genotypic characterization of $L$. pneumophila strains}

Legionella pneumophila Sg1 strains Camperdown 1 (ATCC 43113), Heysham 1 (ATCC 43107) [23], Uppsala 3 [46] and Görlitz 6543 [49] were grown on buffered charcoal yeast extract (BCYE) agar plates (Oxoid, Germany) for $48 \mathrm{hr}$ at $37^{\circ} \mathrm{C}$ under a $5 \% \mathrm{CO}_{2}$ atmosphere. Monoclonal subgrouping was accomplished using the Dresden panel of $\mathrm{mAb}$ as described elsewhere $[13,16]$.

\section{DNA extraction and sequence generation}

DNA was extracted using the EZ1 DNA Tissue Kit (Qiagen, Germany). Prior to sequencing DNA fragments of the LPS-biosynthesis locus were PCR-amplified using GoTaq polymerase (Promega, US-WI) and LPS-specific primers (Additional file 2: Table S1) which were designed based on published L. pneumophila genomes. Initial denaturation was carried out at $95^{\circ} \mathrm{C}$ for $2 \mathrm{~min}$ followed by $30-35$ cycles: $95^{\circ} \mathrm{C}$ denaturation for $30 \mathrm{~s}$, annealing at various temperatures for $1 \mathrm{~min}$ and elongation at $72^{\circ} \mathrm{C}$ for $1 \mathrm{~min} / \mathrm{kb}$. Final elongation for $5 \mathrm{~min}$ at $72^{\circ} \mathrm{C}$ completed the amplification protocol. The PCR result was checked on $1.5 \%$ agarose gel with $5 \mathrm{~V} / \mathrm{cm}$ (LE Agarose, Biozym, Germany) and purified (MSB Spin PCRapace, Invitek, Germany) for sequence reaction.

Sequencing reactions were accomplished by a cyclesequencing procedure on an automated DNA sequencing machine (ABI Prism 377, Applied Biosystems, US-CA).

The LPS-biosynthesis locus of the strain L10/23 was sequenced during a whole genome sequencing project. This strain was isolated during a cooling tower related outbreak in Ulm (Germany) in 2010 [53].

\section{Sequence annotation and analysis}

Obtained sequences of Camperdown 1, Heysham 1, Uppsala 3, Görlitz 6543 and L10/23 were assembled using SeqMan (DNASTAR Lasergene 8, US-WI) and controlled against public databases using BLAST [54]. ORF annotation of all analyzed strains was accomplished with GeneMark.hmm [55] and Artemis [56]. The annotation and the numbering of ORFs was based on the 
initially described LPS-biosynthesis locus of strain RC1 (mAb-subgroup OLDA) [21] and if possible supplemented by further description of genes, gene products and their putative functions using BLAST, BLASTP [54,57], the LegionellaScope database of the MicroScope Microbial Genome Annotation Platform [58] and the Conserved Domain Database [59]. Since Lüneberg et al. analyzed the strain $\mathrm{RC} 1$ which had 30 ORFs the numbering of ORFs in other L. pneumophila Sg1 strains with deviating ORF numbers is not continual [21]. The genes iraA (ORF 29) and iraB (ORF 30) were not taken into account as part of the LPS-biosynthesis locus. Both formed a small 2-gene operon responsible for iron assimilation, infection and virulence [60].

The putative coding regions were compared to already known LPS-biosynthesis ORFs of published $L$ pneumophila strains using the SeqMan program. The LPSbiosynthesis clusters of the strains were deposited in the EMBL database under the number [EMBL: HE980447] for strain Camperdown 1 (mAb-subgroup Camperdo wn), [EMBL: HE980446] for strain Heysham 1 (mAbsubgroup Heysham), [EMBL: HE980445] for strain Uppsala 3 (mAb-subgroup Knoxville), [EMBL: HF678227] for strain Görlitz 6543 (mAb-subgroup Bellingham) and [EMBL: HF545881] for strain L10/23 (mAb-subgroup Knoxville) (Table 2).

Sequence homologies of single ORFs were calculated based on multiple alignments using BioNumerics 6.0 (Applied Maths NV, Belgium) and BLASTP [57]. Cluster analysis was performed using the UPGMA method of the BioNumerics 6.0 software package.

The sequences of other LPS-biosynthesis loci were obtained from complete genomes of the following strains: Paris (mAb-subgroup Philadelphia) (GenBank: NC_006368.1), Lens (mAb-subgroup Benidorm) (Gen Bank: NC_006369.1), Philadelphia 1 (mAb-subgroup Philadelphia) (GenBank: NC_002942.5), Alcoy 2300/99 (mAb-subgroup Knoxville) (GenBank: NC_014125.1), Corby (mAb-subgroup Knoxville) (GenBank: NC_0094 94.2), Lorraine (mAb-subgroup Allentown) (EMBL: FQ958210), HL 06041035 (mAb-subgroup Bellingham) (EMBL: FQ958211), RC1 (mAb-subgroup OLDA) (EMBL: AJ277755) and 130b (mAb-subgroup Benidorm) (EMBL: FR687201.1) (Table 2) [21,28,29,31-34]. Since the genome of $130 \mathrm{~b}$ is a draft version we closed a sequencing gap in scaffold 4 (position 918107 to 918206) using PCR and sequencing.

\section{Availability of supporting data}

The data sets supporting the results of this article are available in the LabArchives repository, DOI:10.6070/ H4WM1BBQ. It includes a list of all primers used for ORF amplification and sequence generation (Additional file 2: Table S1), a spreadsheet containing detailed information about the LPS-biosynthesis locus such as ORF identifier, ORF size and putative size of the translated ORF product (Additional file 1: Table S2) as well as the \% GC content of the ORFs of the Sg1-specific region (Additional file 1: Table S3).

\section{Additional files}

\section{Additional file 1: This file contains two spreadsheets containing the supporting data S2 and S3. Table S2. Genes/proteins of the LPS- biosynthesis locus of L. pneumophila Sg1 strains. Table S3. Percentage GC-content of single ORFs, regions and the whole LPS-biosynthesis loci of L. pneumophila Sg1 strains.}

Additional file 2: Table S1. This document summarizes all primers used for amplification of LPS-biosynthesis ORFs and sequence generation.

\section{Abbreviations}

LPS: Lipopolysaccharide; mAb: Monoclonal antibody; ORF: Open reading frame; Sg1: Serogroup 1; GlcNAc: N-acetyl-glucosamine; QuiNAc: N-acetyl-quinovo samine; UDP-Bac2Ac4Ac: UDP-N,N'-diacetylbacillosamine; CMP-Leg5Ac7Ac: CMP5,7-diacetamido-3,5,7,9-tetradeoxy-D-glycero-D-talo-nonulosonic acid; CMPLeg5Am7Ac: CMP-5-acetamidino-7-acetamido-3,5,7,9-tetradeoxy-D-glycero-D-talononulosonic acid; CMP-Leg5AmNMe7Ac: CMP-5-acetimidoyl (N-methyl) amino-7 -acetamido-3,5,7,9-tetradeoxynon-2-ulosonic acid.

\section{Competing interests}

The authors declare that they have no competing interests.

\section{Authors' contributions}

MP generated sequences of strains Camperdown 1 and Heysham 1 conducted comparative genetic and phylogenic studies, interpreted the results and drafted the manuscript. AT and SM generated sequences of strains Uppsala 3 and Görlitz 6543. KH generated the genome sequence of strain L10/23. JWM reviewed the manuscript. CL conceived and supervised the work, assisted with inspiring discussions and ideas, helped interpreting the results and reviewed the manuscript. All authors read and approved the manuscript.

\section{Acknowledgement}

We thank Sigrid Gäbler, Kerstin Lück and Ines Wolf for technical assistance. This work was partly supported by the Robert Koch-Institute grant 1369-364 to CL. Dedicated to the memory of Dr. Jürgen Helbig, Dresden, Germany.

\section{Author details}

${ }^{1}$ Institute of Medical Microbiology and Hygiene, Faculty of Medicine 'Carl Gustav Carus', University of Technology Dresden, Fetscherstraße 74, Dresden D-01307, Germany. ²Department of Medical Microbiology, Radboud University Nijmegen Medical Centre, PO Box 9101, Nijmegen, Netherlands. ${ }^{3}$ Cellular Interactions of Bacterial Pathogens, Centre for Biological Security, Division 2 (ZBS2), Robert Koch-Institute, Nordufer 20, Berlin D-13353, Germany.

Received: 24 April 2013 Accepted: 31 August 2013 Published: 4 September 2013

\section{References}

1. Pearce M, Theodoropoulos N, Mandel M, Brown E, Reed K, Cianciotto N: Legionella cardiaca sp. nov., isolated from a case of native valve endocarditis in a human heart. Int J Syst Evol Microbiol 2012, 62:2946-2954.

2. Rowbotham TJ: Preliminary report on the pathogenicity of Legionella pneumophila for freshwater and soil amoebae. J Clin Pathol 1980, 33:1179-1183.

3. Fields BS, Benson RF, Besser RE: Legionella and Legionnaires disease: 25 years of investigation. Clin Microbiol Rev 2002, 15:506-526.

4. Declerck P: Biofilms: the environmental playground of Legionella pneumophila. Environ Microbiol 2010, 12:557-566.

5. Stewart $C R$, Muthye $V$, Cianciotto NP: Legionella pneumophila persists within biofilms formed by Klebsiella pneumoniae, Flavobacterium sp., 
and Pseudomonas fluorescens under dynamic flow conditions. PLOS ONE 2012, 7:e50560.

6. Fraser DW: Legionellosis: evidence of airborne transmission. Ann NY Acad Sci 1980, 353:61-66.

7. Isberg RR, Tj OC, Heidtman M: The Legionella pneumophila replication vacuole: making a cosy niche inside host cells. Nat Rev Microbiol 2009, 7:13-24

8. McDade JE, Shepard CC, Fraser DW, Tsai TR, Redus MA, Dowdle WR: Legionnaires' disease: isolation of a bacterium and demonstration of its role in other respiratory disease. N Engl J Med 1977, 297:1197-1203.

9. Harrison TG, Afshar B, Doshi N, Fry NK, Lee JV: Distribution of Legionella pneumophila serogroups, monoclonal antibody subgroups and DNA sequence types in recent clinical and environmental isolates from England and Wales (2000-2008). Eur J Clin Microbiol Infect Dis 2009, 28:781-791.

10. Joseph CA, Ricketts KD, Yadav R, Patel S: Travel-associated Legionnaires' disease in Europe in 2009. Euro Surveill 2010, 15:5-11.

11. Ciesielski CA, Blaser MJ, Wang WL: Serogroup specificity of Legionella pneumophila is related to lipopolysaccharide characteristics. Infect Immun 1986, 51:397-404

12. Helbig JH, Jacobs $E$, Lück C: Legionella pneumophila urinary antigen subtyping using monoclonal antibodies as a tool for epidemiological investigations. Eur J Clin Microbiol Infect Dis 2012, 31:1673-1677.

13. Helbig JH, Kurtz JB, Pastoris MC, Pelaz C, Lück C: Antigenic lipopolysaccharide components of Legionella pneumophila recognized by monoclonal antibodies: possibilities and limitations for division of the species into serogroups. J Clin Microbiol 1997, 35:2841-2845.

14. Helbig JH, Benson RF, Pelaz C, Jacobs E, Lück C: Identification and serotyping of atypical Legionella pneumophila strains isolated from human and environmental sources. J App/ Microbiol 2007, 102:100-105.

15. Joly JR, Chen $Y Y$, Ramsay D: Serogrouping and subtyping of Legionella pneumophila with monoclonal antibodies. J Clin Microbiol 1983, 18:1040-1046.

16. Helbig JH, Bernander S, Castellani Pastoris M, Etienne J, Gaia V, Lauwers S, Lindsay D, Lück C, Marques T, Mentula S, et al: Pan-European study on culture-proven Legionnaires' disease: distribution of Legionella pneumophila serogroups and monoclonal subgroups. Eur J Clin Microbiol Infect Dis 2002, 21:710-716

17. Knirel YA, Valvano MA: Bacterial Lipopolysaccharides: Structure, Chemical Synthesis, Biogenisis and Interaction with Host Cells. Vienna: Springer Vienna; 2011.

18. Knirel YA, Rietschel ET, Marre R, Zähringer U: The structure of the O-specific chain of Legionella pneumophila serogroup 1 lipopolysaccharide. Eur J Biochem 1994, 221:239-245.

19. Zähringer U, Knirel YA, Lindner B, Helbig JH, Sonesson A, Marre R, Rietschel ET: The lipopolysaccharide of Legionella pneumophila serogroup 1 (strain Philadelphia 1): chemical structure and biological significance. Prog Clin Biol Res 1995, 392:113-139.

20. Kooistra O, Herfurth L, Lüneberg E, Frosch M, Peters T, Zähringer U: Epitope mapping of the $\mathrm{O}$-chain polysaccharide of Legionella pneumophila serogroup 1 lipopolysaccharide by saturation-transfer-difference NMR spectroscopy. Eur J Biochem 2002, 269:573-582.

21. Lüneberg E, Zetzmann N, Alber D, Knirel YA, Kooistra $O$, Zähringer U, Frosch $\mathrm{M}$ : Cloning and functional characterization of a $30 \mathrm{~kb}$ gene locus required for lipopolysaccharide biosynthesis in Legionella pneumophila. Int J Med Microbiol 2000, 290:37-49.

22. Zou CH, Knirel YA, Helbig JH, Zähringer U, Mintz CS: Molecular cloning and characterization of a locus responsible for $\mathrm{O}$ acetylation of the $\mathrm{O}$ polysaccharide of Legionella pneumophila serogroup 1 lipopolysaccharide. J Bacterio/ 1999, 181:4137-4141.

23. Joly JR, McKinney RM, Tobin JO, Bibb WF, Watkins ID, Ramsay D: Development of a standardized subgrouping scheme for Legionella pneumophila serogroup 1 using monoclonal antibodies. J Clin Microbio/ 1986, 23:768-771.

24. Helbig JH, Lück PC, Knirel YA, Witzleb W, Zähringer U: Molecular characterization of a virulence-associated epitope on the lipopolysaccharide of Legionella pneumophila serogroup 1. Epidemiol Infect 1995, 115:71-78.

25. Amemura-Maekawa J, Kikukawa K, Helbig JH, Kaneko S, Suzuki-Hashimoto A, Furuhata K, Chang B, Murai M, Ichinose M, Ohnishi M, et al: Distribution of monoclonal antibody subgroups and sequence-based types among Legionella pneumophila serogroup 1 isolates derived from cooling tower water, bathwater, and soil in Japan. Appl Environ Microbiol 2012, 78:4263-4270.
26. Harrison TG, Doshi N, Fry NK, Joseph CA: Comparison of clinical and environmental isolates of Legionella pneumophila obtained in the UK over 19 years. Clin Microbiol Infect 2007, 13:78-85.

27. Reimer AR, Au S, Schindle S, Bernard KA: Legionella pneumophila monoclonal antibody subgroups and DNA sequence types isolated in Canada between 1981 and 2009: Laboratory Component of National Surveillance. Eur J Clin Microbiol Infect Dis 2010, 29:191-205.

28. D'Auria D, Jimnez-Hernndez N, Peris-Bondia F, Moya A, Latorre A: Legionella pneumophila pangenome reveals strain-specific virulence factors. BMC Genomics 2010, 11:181.

29. Glöckner G, Albert-Weissenberger C, Weinmann E, Jacobi S, Schunder E, Steinert M, Hacker J, Heuner K: Identification and characterization of a new conjugation/type IVA secretion system (trb/tra) of Legionella pneumophila Corby localized on two mobile genomic islands. Int J Med Microbiol 2008, 298:411-428.

30. Cazalet C, Rusniok C, Brüggemann H, Zidane N, Magnier A, Ma L, Tichit M, Jarraud S, Bouchier C, Vandenesch F, et al: Evidence in the Legionella pneumophila genome for exploitation of host cell functions and high genome plasticity. Nat Genet 2004, 36:1165-1173.

31. Chien M, Morozova I, Shi S, Sheng H, Chen J, Gomez SM, Asamani G, Hill K, Nuara J, Feder $M$, et al: The genomic sequence of the accidental pathogen Legionella pneumophila. Science 2004, 305:1966-1968.

32. Gomez-Valero L, Rusniok C, Jarraud S, Vacherie B, Rouy Z, Barbe V, Medigue C, Etienne J, Buchrieser C: Extensive recombination events and horizontal gene transfer shaped the Legionella pneumophila genomes.

BMC Genomics 2011, 12:536.

33. Schroeder GN, Petty NK, Mousnier A, Harding CR, Vogrin AJ, Wee B, Fry NK, Harrison TG, Newton HJ, Thomson NR, et al: Legionella pneumophila strain $130 \mathrm{~b}$ possesses a unique combination of type IV secretion systems and novel Dot/lcm secretion system effector proteins. J Bacterio/ 2010, 192:6001-6016.

34. Cazalet C, Jarraud S, Ghavi-Helm Y, Kunst F, Glaser P, Etienne J, Buchrieser C: Multigenome analysis identifies a worldwide distributed epidemic Legionella pneumophila clone that emerged within a highly diverse species. Genome Res 2008, 18:431-441.

35. Merault N, Rusniok C, Jarraud S, Gomez-Valero L, Cazalet C, Marin M, Brachet E, Aegerter P, Gaillard JL, Etienne J, et al: Specific Real-Time PCR for simultaneous detection and identification of Legionella pneumophila serogroup 1 in water and clinical samples. Appl Environ Microbiol 2011 77:1708-1717

36. Glaze PA, Watson DC, Young NM, Tanner ME: Biosynthesis of CMP-N, $\mathrm{N}$-diacetyllegionaminic acid from UDP-N, N -diacetylbacillosamine in Legionella pneumophila. Biochemistry 2008, 47:3272-3282.

37. Schoenhofen IC, McNally DJ, Vinogradov E, Whitfield D, Young NM, Dick S, Wakarchuk WW, Brisson J-R, Logan SM: Functional characterization of dehydratase/aminotransferase pairs from Helicobacter and Campylobacter: Enzymes distinguishing the pseudaminic acid and bacillosamine biosynthetic pathways. J Biol Chem 2006, 281:723-732

38. Knirel YA, Shashkov AS, Tsvetkov YE, Jansson P-E, Zähringer U: 5,7-diamino3,5,7,9-tetradeoxynon-2-ulosonic acids in bacterial glycopolymers: Chemistry and biochemistry. Adv Carbohydr Chem Biochem 2003, 58:371-417.

39. Lewis AL, Hensler ME, Varki A, Nizet V: The group B streptococcal sialic acid O-acetyltransferase is encoded by neuD, a conserved component of bacterial sialic acid biosynthetic gene clusters. J Biol Chem 2006, 281:11186-11192.

40. McNally DJ, Aubry AJ, Hui JPM, Khieu NH, Whitfield D, Ewing CP, Guerry P, Brisson J-R, Logan SM, Soo EC: Targeted metabolomics analysis of Campylobacter coli VC167 reveals legionaminic acid derivatives as novel flagellar glycans. J Biol Chem 2007, 282:14463-14475.

41. Knirel YA, Senchenkova SN, Kocharova NA, Shashkov AS, Helbig JH, Zähringer U: Identification of a homopolymer of 5-acetamidino-7acetamido-3,5,7,9-tetradeoxy-D-glycero-D-talo-nonulosonic acid in the lipopolysaccharides of Legionella pneumophila Non-1 serogroups. Biochemistry (Mosc) 2001, 66:1035-1041.

42. Sahr T, Rusniok C, Dervins-Ravault D, Sismeiro O, Coppee J-Y, Buchrieser C: Deep sequencing defines the transcriptional map of $L$. pneumophila and identifies growth phase-dependent regulated ncRNAs implicated in virulence. RNA Biol 2012, 9:503-519.

43. Farhat C, Mentasti M, Jacobs E, Fry NK, Lück C: The N-Acylneuraminate cytidyltransferase gene, neuA, is heterogenous in Legionella pneumophila strains but can be used as a marker for epidemiological 
typing in the consensus sequence-based typing scheme. J Clin Microbiol 2011, 49:4052-4058.

44. Ledesma E, Camaró ML, Carbonell E, Sacristan T, Marti A, Pellicer S, Llorca J, Herrero P, Dasi MA: Subtyping of Legionella pneumophila isolates by arbitrarily primed polymerase chain reaction. Can J Microbiol 1995, 41:846-848.

45. Kozak NA, Benson RF, Brown E, Alexander NT, Taylor TH, Shelton BG, Fields BS: Distribution of lag-1 alleles and sequence-based types among Legionella pneumophila serogroup 1 clinical and environmental isolates in the United States. J Clin Microbiol 2009, 47:2525-2535

46. Bernander S, Jacobson K, Helbig JH, Lück C, Lundholm M: A hospitalassociated outbreak of Legionnaires disease caused by Legionella pneumophila serogroup 1 is characterized by stable genetic fingerprinting but variable monoclonal antibody patterns. J Clin Microbiol 2003, 41:2503-2508.

47. Lück C, Freier T, Steudel C, Knirel YA, Lüneberg E, Zähringer U, Helbig JH: A point mutation in the active site of Legionella pneumophila $\mathrm{O}$-acetyltransferase results in modified lipopolysaccharide but does not influence virulence. Int J Med Microbiol 2001, 291:345-352

48. Wagner C, Krönert C, Lück C, Jacobs E, Cianciotto NP, Helbig JH: Random mutagenesis of Legionella pneumophila reveals genes associated with lipopolysaccharide synthesis and recognition by typing monoclonal antibodies. J Appl Microbiol 2007, 103:1975-1982.

49. Thürmer A, Helbig JH, Jacobs E, Lück C: PCR-based 'serotyping' of Legionella pneumophila. J Med Microbiol 2009, 58:588-595.

50. Greenfield LK, Whitfield C: Synthesis of lipopolysaccharide O-antigens by ABC transporter-dependent pathways. Carbohydr Res 2012, 356:12-24.

51. Price MN, Huang KH, Alm EJ, Arkin AP: A novel method for accurate operon predictions in all sequenced prokaryotes. Nucleic Acids Res 2005, 33:880-892.

52. Kooistra O, Lüneberg E, Knirel YA, Frosch M, Zähringer U: N-Methylation in polylegionaminic acid is associated with the phase-variable epitope of Legionella pneumophila serogroup 1 lipopolysaccharide. Identification of 5-(N, N-dimethylacetimidoyl)amino and 5-acetimidoyl(N-methyl) amino-7-acetamido-3,5,7,9-tetradeoxynon-2-ulosonic acid in the O-chain polysaccharide. Eur J Biochem 2002, 269:560-572.

53. von Baum H, Härter G, Essig A, Lück C, Gonser T. Embacher A, Brockmann S. Preliminary report: outbreak of Legionnaires disease in the cities of Ulm and Neu-Ulm in Germany, December 2009 - January 2010. Euro Surveill 2010, 15:19472.

54. Altschul SF, Madden TL, Schäffer AA, Zhang J, Zhang Z, Miller W, Lipman DJ: Gapped BLAST and PSI-BLAST: a new generation of protein database search programs. Nucleic Acids Res 1997, 25:3389-3402.

55. Lukashin A, Borodovsky M: GeneMark.hmm: new solutions for gene finding. Nucleic Acids Res 1998, 26:1107-1115.

56. Rutherford K, Parkhill J, Crook J, Horsnell T, Rice P, Rajandream MA, Barrell B: Artemis: sequence visualization and annotation. Bioinformatics 2000, 16:944-945.

57. Altschul SF, Wootton JC, Gertz EM, Agarwala R, Morgulis A, Schäffer AA, Yu Y-K: Protein database searches using compositionally adjusted substitution matrices. FEBS J 2005, 272:5101-5109.

58. Vallenet D, Engelen S, Mornico D, Cruveiller S, Fleury L, Lajus A, Rouy Z, Roche D, Salvignol G, Scarpelli C, Médigue C: MicroScope: a platform for microbial genome annotation and comparative genomics. Database 2009, 2009:Bap21.

59. Marchler-Bauer A, Lu S, Anderson JB, Chitsaz F, Derbyshire MK, DeWeeseScott C, Fong JH, Geer LY, Geer RC, Gonzales NR, et al: CDD: a Conserved Domain Database for the functional annotation of proteins. Nucleic Acids Res 2011, 39:D225-D229.

60. Viswanathan VK, Edelstein PH, Pope CD, Cianciotto NP: The Legionella pneumophila ira $A B$ locus is required for iron assimilation, intracellular infection, and virulence. Infect Immun 2000, 68:1069-1079.

doi:10.1186/1471-2180-13-198

Cite this article as: Petzold et al: A structural comparison of lipopolysaccharide biosynthesis loci of Legionella pneumophila serogroup 1 strains. BMC Microbiology 2013 13:198.

\section{Submit your next manuscript to BioMed Central and take full advantage of:}

- Convenient online submission

- Thorough peer review

- No space constraints or color figure charges

- Immediate publication on acceptance

- Inclusion in PubMed, CAS, Scopus and Google Scholar

- Research which is freely available for redistribution 